\title{
Investigating the Potential of Using Quota Count as a Design Metric
}

\author{
Peter Hollingsworth* and David Sulitzer ${ }^{\dagger}$ \\ The University of Manchester, Manchester, M13 9PL, UK
}

\begin{abstract}
Identifying easy to use and communicate extensive design metrics for aircraft noise could be of substantial benefit to the aircraft design community. The current means of communication often revolves around margin to the certification stringencies. These stringencies are hard to communicate and not directly related to external impact. A good design metric correlates well with the individual aircraft's effect on these broader measures. This paper proposes the use of Quota Count, which was devised as a means of limiting night-time exposure to single equivalent events at London area airports. It is directly related to the actual energy emitted by the an aircraft and the resulting time average values observed on the ground. It is straightforward to communicate and comprehend. It is based upon the certification noise values, and therefore, straightforward for a design engineer to model. This paper answers the question does Quota Count relate well with cumulative measures especially day-night noise level estimates of the area exposed to a given cumulative level? Given that Quota Count, and a simple cumulative Quota Count model correlate highly with these measures, it is reasonable to suggest that Quota Count be used as a primary noise metric for new designs.
\end{abstract}

\section{Nomenclature}

A Linear transformation, additive coefficient

B Linear transformation, multiplicative coefficient

*Lecturer, School of Mechanical, Aerospace and Civil Engineering, Sackville Street, Manchester, UK, peter.hollingsworth@manchester.ac.uk, AIAA Member

${ }^{\dagger}$ Undergraduate Student, School of Mechanical, Aerospace and Civil Engineering, Sackville Street, Manchester, UK , david.sulitzer@student.manchester.ac.uk 


\begin{tabular}{ll}
$\mathrm{g}_{\mathrm{i}}$ & Event weighting factor \\
$\mathrm{L}_{\mathrm{DN}}$ & Day-Night noise level, similar to $\mathrm{L}_{\text {eq }}$ except with a 10dB night penalty, dBA \\
$\overline{\mathrm{L}}_{\mathrm{E}}$ & Operations averaged equivalent noise level, in dBA \\
$\mathrm{L}_{\mathrm{eq}}$ & Time-averaged equivalent noise level in dBA \\
$\mathrm{L}(\mathrm{t})$ & Noise time history, in dBA \\
$\mathrm{P}$ & Passenger aircraft daytime regression coefficient \\
$\mathrm{Q}$ & Passenger aircraft nighttime regression coefficient \\
$\mathrm{R}$ & Cargo aircraft daytime regression coefficient \\
$\mathrm{S}$ & Cargo aircraft night regression coefficient \\
$\mathrm{t}_{0}$ & Event duration, in seconds \\
$\mathrm{T}_{0}$ & Multi-event time average, in seconds \\
$\mathrm{X}_{\mathrm{i}}$ & Number of operations at airport i \\
$\Delta_{\mathrm{i}}$ & log based event weighting factor, see $\mathrm{g}_{\mathrm{i}}$ \\
$\delta$ & Daytime operations ratio \\
$\mathrm{a}$ & Aircraft class \\
$\mathrm{AP}$ & Approach \\
arr & Arrival \\
best & Best-in-class \\
car & Cargo aircraft \\
cont & Continuous \\
cum & Cumulative \\
dep & Departure \\
$\mathrm{E}$ & Single-event time average equivalent \\
$\mathrm{e}$, eq & Multi-event time-averaged equivalent \\
$\mathrm{FO}$ & Flyover \\
$\mathrm{i}$ & Airport index, event index \\
lim & Certification limit, in EPNdB \\
pax & Passenger aircraft \\
rep & Representative aircraft \\
$\mathrm{SL}$ & Sideline \\
$\mathrm{TO}$ & Takeoff \\
& \\
\hline
\end{tabular}

\section{Introduction}

The development of a quick and relatively easy means of evaluating the value ${ }^{1,2}$ of future aircraft and aircraft system designs is predicated, among other things, on arriving at a 
series of relatively straightforward ways of trading between a range of metrics including environmental impact. The problem with this is that the current certification stringencies do not provide an easy to understand and easy to implement means of trading different impacts. This stems from the fact that it is often difficult to translate between an aircraft's emissions or noise and the overall environmental impact of the fleet. However, there is a significant body of work that is aimed at addressing single aircraft to fleet level impacts. ${ }^{3-7}$ Unfortunately, even with these linkages it will be necessary for the design engineer to meet the existing and future certification requirements. This presents a conundrum, are there commonly used engineering or marketing metrics that are both easy to communicate and map well with both single event and fleet level impacts?

The current means of reporting environmental performance of new aircraft concepts is typically the margin to the relevant ICAO/CAEP standard. This leaves significant room for confusion as these standards are purposely divorced from direct emissions and noise impacts, in the case of aircraft noise these limits are a function of maximum weight, and in some cases the number of engines. ${ }^{8,9}$ Beyond the certification metrics there exists a body both aircraft and fleet level metrics that potentially provide more insight into the design choices. This is especially true in the area of aircraft noise. An aircraft noise metric exists that is designed to map directly to local noise exposure, the Quota Count ${ }^{10}$ (QC) system that governs airport operations at UK airports.

The QC system is designed to provide a mean of estimating single operation effective noise impact, in this case A weighted Sound Exposure Level (SEL) and the area exposed to a specific SEL. This equivalent noise metric is commonly used to estimate disturbance ${ }^{11}$ and awakenings. ${ }^{12}$ Additionally, QC has an advantage over certification noise or margin to a certification limit in that is easier to communicate to the nontechnical public. The potential weakness with using $\mathrm{QC}$ as the primary metric is that there exists a range of other metrics, specifically the cumulative metrics, ${ }^{11}$ of which Day-Night Noise Level (DNL) is one, that QC was not specifically designed to address.

Ideally, it would be possible to find a relationship between $\mathrm{QC}$ and all current and potential future measures of noise impact. This includes both the common $A$ weighted and more general broadband measures. However, since the current regulatory and impact guidance often focuses on both single event and cumulative equivalent noise this paper only investigates how well $\mathrm{QC}$ correlates with models that estimate the most commonly used cumulative noise measures. In doing this it is useful to look at what quick to evaluate noise measures are available to the engineer, as opposed to the more detailed and sophisticated analysis tools such as $\mathrm{INM}^{13}$ and ANCON. ${ }^{14}$ The most well documented and easiest to use of these is the Noise Energy Equivalent (NEE) method described by Dikshit and Crossley, ${ }^{4}$ which is designed to map changes in aircraft technology levels to fleet level noise impacts. 
Specifically, the area exposed to $\geq 65 \mathrm{~dB}$ DNL noise. This paper investigates the applicability of using QC to represent single event metrics, the ability of $\mathrm{QC}$ and specifically a $\mathrm{QC}$ derived cumulative model to match the results obtained by the current cumulative analysis methods. The result of which would allow for a simple, straightforward additive model by which to compare the impact of different design options across a range of single event and cumulative noise impacts. Further, because QC is based the noise certification levels, it is well within the modelling and simulation capability of modern aircraft and engine design organizations. Further, the linkage between certification noise and design variables is dependent upon the specific organisations and their analysis tools. As such it is beyond the scope of this paper

\section{The Quota Count System}

The Quota Count (QC) system is recognized as a practical and straightforward means of classifying the noise impact of arriving and departing aircraft around an airport. It was first introduced in the 1990s based on a 1991 noise analysis of commercial aircraft operating at United Kingdom airports. ${ }^{15,16}$ The current QC classifications ${ }^{17}$ have been developed to insure that the noise impact around an airport is correctly quantified for each individual aircraft operation. Two aspects are considered for the adverse effects of noise: the area of exposure and the number of people affected. The QC System derives from the official certificated noise levels. The reasons for this is that those are generally considered to be reliable indicators of aircraft noise performance, they are available for vast majority of civil transport aircraft operating in the Western world, and are openly published.

The Quota Count uses the standard certification noise levels, ${ }^{17}$ and derives the QC classification from a combination of three reference points: the approach, under the descent path $2000 \mathrm{~m}$ before landing threshold; the lateral or sideline, at the point where noise is greatest on a line $450 \mathrm{~m}$ to the side of the initial climb after lift-off; and the flyover, under the departure climb path, $6500 m$ from Start-of-Roll. Those reference points can be seen on Figure 1. The QC Classification goes from 0.25 to 16 , with a few aircraft below the 0.25 level considered exempt, and are related to ranges of EPNL values either for departure or for arrivals. Table 1 lists the QC Classification for each range of EPNL values expressed in EPNdB. Equations 1 and 2 present the method for calculating the relevant EPNL values at take-off and landing using the EPNL values recorded at the three certification reference points. ${ }^{17}$

$$
\begin{gathered}
E P N L_{T O, Q C}=\frac{E P N L_{\text {Flyover }}+E P N L_{\text {Sideline }}}{2} \\
E P N L_{\text {Land,QC }}=E P N L_{\text {Approach }}-9 d B
\end{gathered}
$$




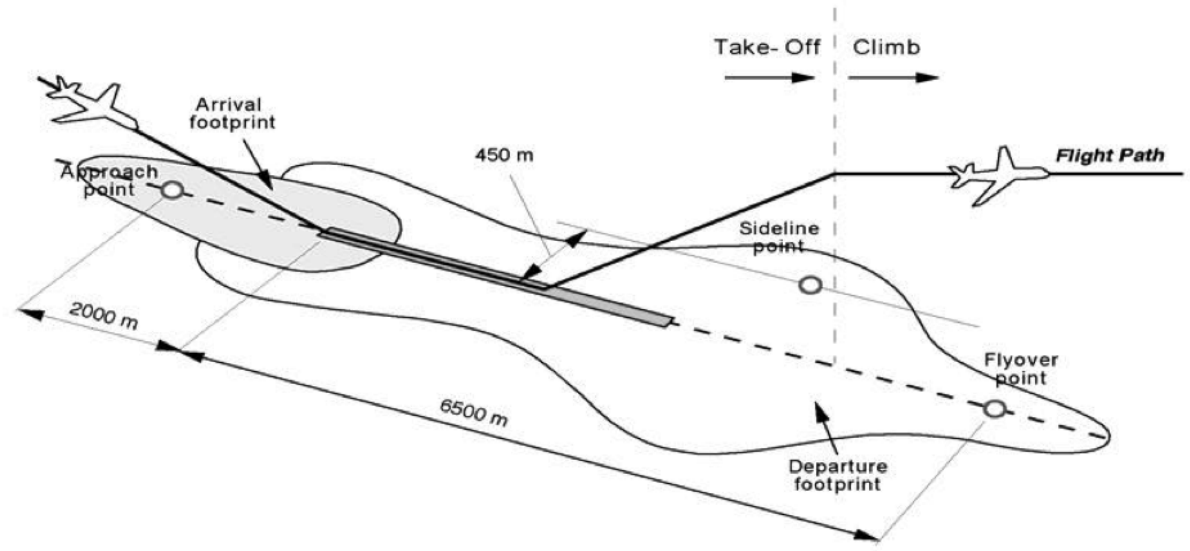

Figure 1. Aircraft Noise Certification Measurement Points ${ }^{17}$

Table 1. Quota Count Classification System ${ }^{17}$

\begin{tabular}{ll}
\hline \hline Qualifying Level (EPNdB) & QC Classification \\
\hline$\geq 102$ & 16 \\
$99-101.9$ & 8 \\
$96-98.9$ & 4 \\
$93-95.9$ & 2 \\
$90-92.9$ & 1 \\
$87-89.9$ & 0.5 \\
$84-86.9$ & 0.25 \\
$<84$ & Exempt \\
\hline \hline
\end{tabular}


The values of EPNL for departures and for arrivals are calculated differently. Departure values are equal to the average of the lateral EPNL and the flyover EPNL while arrival ones are based on the approach $E P N L_{A P}-9$. It is necessary to reduce the EPNL value found for the approach reference point by $9 \mathrm{~dB}$ because the noise from a departing aircraft travels between three and five times as far as the one from an arriving aircraft before reaching the reference points. This means that to correlate well with the 90dB SEL contour area the approach certification level had to be reduced accordingly.

\section{Noise Energy Equivalent Model}

In work for NASA Langley Research Center Diskhit and Crossley ${ }^{4}$ attempted to create a noise model that could account for different fleets of aircraft at different airports. The model needed to be single valued, rapidly computable, have a simple formulation, be correlated with a standard noise model, and be flexible in order to accept technological evolutions. ${ }^{4}$ The resulting method and metric meets these requirements and gives a quick measure of "Noise Area" impacted by noise for any airports from only a few inputs. The resulting model closely approximats the Day-Night Level ${ }^{4}$ (DNL), which is the standard noise metric used at airports to account for the maximum acceptable noise exposure for the people living within the area. More precisely, it is the $65 \mathrm{~dB}$ DNL limit that is utilized when monitoring the noise areas around an airport. The Integrated Noise Model $^{13}$ (INM) is recommended by the Federal Aviation Administration to compute DNL contours. The results obtained by using the model correlated with INM values which demonstrates the utility and accuracy of the model.

Because of the logarithmic nature of EPNL the authors used NEE instead of EPNL values for their calculations. The NEE value, which is related to EPNL can be calculated using Equation (3): ${ }^{4}$

$$
N E E=10^{\left(\frac{E P N L}{10}\right)}+C
$$

In order to simplify their fleet level analysis Dikshit and Crossley modelled a generic fleet ${ }^{4}$ of aircraft with characteristics that could represent the entire American fleet. The decision was made to differentiate classes of passenger aircraft based on their seating capacity since their noise energy is highly correlated with aircraft size. Furthermore, for each class of passenger aircraft, a "Representative" and a "Best-in-Class" aircraft were selected. Dikshit

and Crossley select the aircraft with the largest number of operations in each seat class, in 2005, to populate the "Representative" aircraft table. Further, the "best-in-class" aircraft were those with the most recent Entry-in-Service (EIS) date. The same rules were applied to classify cargo aircraft. In addition, three airport configurations were taken into account. Using these aircraft to represent operations at an airport the cumulative noise exposure area 
is estimated using linear summation, shown in Equation (4): ${ }^{4}$

$$
\begin{aligned}
& \text { Area }_{i}=\sum_{a=1}^{6}\left[\left\{\left(P_{a} \delta_{i}^{T O}+Q_{a}\left(1-\delta_{i}^{T O}\right)\right)\left(N E E_{a, r e p}^{\text {pax }, T O} X_{a, i, T O}^{\text {pax,rep }}+N E E_{a, b e s t}^{\text {pax } T O} X_{a, i, T O}^{\text {pax }, \text { best }}\right)\right.\right.
\end{aligned}
$$

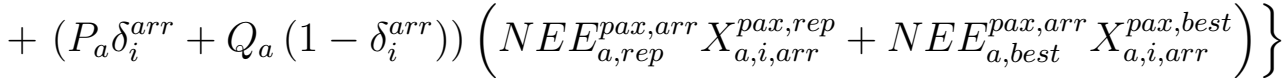

$$
\begin{aligned}
& +\left\{\left(R_{a} \delta_{i}^{T O}+S_{a}\left(1-\delta_{i}^{T O}\right)\right)\left(N E E_{a, r e p}^{c a r, T O} X_{a, i, T O}^{c a r, r e p}+N E E_{a, b e s t}^{c a r, T O} X_{a, i, T O}^{c a r, b e s t}\right)\right. \\
& \left.\left.+\left(R_{a} \delta_{i}^{a r r}+S_{a}\left(1-\delta_{i}^{a r r}\right)\right)\left(N E E_{a, \text { rep }}^{\text {car, arr }} X_{a, i, a r r}^{\text {car, rep }}+N E E_{a, \text { best }}^{\text {car }, \text { arr }} X_{a, i, a r r}^{\text {car }, \text { best }}\right)\right\}\right] \frac{1}{10000}
\end{aligned}
$$

Table 2 lists the resulting regression coefficients, used to estimate the area within the $65 \mathrm{~dB}$ DNL contour, while Tables 3 and 4 list the simplified passenger and cargo fleets respectively. ${ }^{4}$

Table 2. NEE Regression Coefficents ${ }^{4}$

\begin{tabular}{ccccc}
\hline \hline Class(a) & $\mathbf{P}_{\mathbf{a}}$ & $\mathbf{Q}_{\mathbf{a}}$ & $\mathbf{R}_{\mathbf{a}}$ & $\mathbf{S}_{\mathbf{a}}$ \\
\hline 1 & 0.7353 & 0.0559 & 0.2272 & 2.7268 \\
2 & 0.2464 & 4.4131 & 1.9546 & 7.2962 \\
3 & 0.2706 & 4.4075 & 2.3337 & 4.1783 \\
4 & 0.8297 & 0.0100 & 0.3663 & 5.5052 \\
5 & 0.0487 & 4.0945 & 2.0928 & 5.1490 \\
6 & 0.2660 & 2.0587 & 0.2963 & 1.8002 \\
\hline \hline
\end{tabular}

Table 3. Represenative and Best-in-class Passenger Aircraft, from Dikshit and Crossley ${ }^{4}$

\begin{tabular}{ccccc}
\hline \hline Class & Seats & Representative & Best-in-class & EIS date \\
\hline Class 1 & $20-50$ & Canadair RJ 200 & Embraer RJ 145 & 19-Dec-96 \\
Class 2 & $51-99$ & Canadair RJ 700 & Embraer RJ 170 & 08-Mar-04 \\
Class 3 & $100-149$ & Boeing 737-300 & Boeing 737-700 & 18-Jan-98 \\
Class 4 & $150-199$ & Boeing 757-200 & Boeing 737-800 & 24-Apr-98 \\
Class 5 & $200-299$ & Boeing 767-300 & Airbus A330-200 & 29-May-98 \\
Class 6 & $300+$ & Boeing 777-200 & Boeing 777-200 & 09-Feb-97 \\
\hline \hline
\end{tabular}

Table 4. Represenative and Best-in-class Cargo Aircraft, from Dikshit and Crossley ${ }^{4}$, Note that the number of seats is used to represent the size of the aircraft

\begin{tabular}{ccccc}
\hline \hline Class & Seats & Representative & Best-in-class & EIS date \\
\hline Class 1 & $20-50$ & Embraer 120 & Canadair RJ 200 & N/A \\
Class 2 & $51-99$ & Boeing 727-100 & Canadair RJ 700 & N/A \\
Class 3 & $100-149$ & Boeing 727-200 & Boeing 737-300 & N/A \\
Class 4 & $150-199$ & Airbus A300-600 & Boeing 757-200 & N/A \\
Class 5 & $200-299$ & Boeing 747-400F-200 & Boeing 777-200 & N/A \\
\hline \hline
\end{tabular}


The NEE area method provides a very easy to implement approximation for the area exposed to the $\geq 65 \mathrm{~dB}$ DNL values around an airport. As such it provides an easier way to quickly measure the effect of new technology introductions. Unfortunately, NEE values are generally very large numbers and can be quite difficult to communicate to lay individuals. Further, Dikshit and Crossley's ${ }^{4}$ work focused on cumulative metrics only and not single event metrics.

\section{Relationship Between QC, Single Event and Cumulative Metrics}

The Quota Count scheme was designed to allow for a quick approximation between the flight of a single aircraft out of one of the three main London area airports and the resulting area exposed to a single even sound exposure level (SEL) above $90 \mathrm{~dB} .{ }^{15-18}$ This was used to set noise impact limits, night time operations restrictions, and ultimately, charges/fees for noise exposures. ${ }^{10,17-19}$ As part of the decision that created and enforced the Quota Count systems it was agreed that the relationship between QC and both the 90dB SEL contour and actual single event noise exposure would be periodically reevaluated. ${ }^{10,19}$ During this reevaluation it was determined that the relationship between QC and the actual noise exposure held up well for the more modern fleet. ${ }^{10,17,19}$ However, it was also determined that the QC levels tended to slightly underpredict the actual noise levels at the monitoring stations around the airport. These underpredictions were fairly consistent across aircraft types. Consequently White et al. ${ }^{19}$ and the Department of Transport ${ }^{17}$ both consider that Quota Count continues to provide a good estimator of actual noise exposure for the entire range of transport aircraft.

Sound Exposure Level is a single event metric, and while SEL is commonly used to measure certain noise impacts, time averaged cumulative impacts are also important. As such any recommendation to use QC as an intermediary, extensive design metric need to explore the relationship between QC and commonly used cumulative metrics, both at an aircraft level and at the fleet level.

\section{IV.A. QC, SEL and Cumulative Metrics}

Cumulative noise metrics are often used as a surrogate for many adverse noise impacts and ultimately for land use planning around airports. Consequently, if QC is going to be recommended as the intermediate noise design metric it needs to correlate well with not just single event metrics such as SEL but also with the cumulative metrics. There is a simple mathematical relationship between single event and generic cumulative measures, shown in 
Equation (5). ${ }^{20}$

$$
L_{e q, W}=10 \log \left[\frac{t_{0}}{T_{0}} \sum_{i=1}^{N} g_{i} 10^{L_{E, i} / 10}\right]+C
$$

where:

$$
L_{E}=10 \log \left[\frac{1}{t_{0}} \int_{t_{1}}^{t_{2}} 10^{L(t) / 10} d t\right]
$$

In the case of $L_{D N}$ the following constants are used: ${ }^{20}$

- $L_{E}=L_{A E}$, A weighted SEL value

- $t_{0}=1 \mathrm{sec}$

- $T_{0}=86,400 \mathrm{sec}$

- $g_{i}=1$ for daytime and $g_{i}=10$ for nighttime

Replacing $g_{i}$ with Equation (7), allows a simpler formulation where SEL levels are modified directly, this is shown in Equation (8). ${ }^{20}$

$$
\begin{gathered}
\Delta_{i}=10 \log \left(g_{i}\right) \\
L_{e q, W}=10 \log \left[\frac{t_{0}}{T_{0}} \sum_{i=1}^{N} 10^{\left(L_{E, i}+\Delta_{i}\right) / 10}\right]+C
\end{gathered}
$$

In the case of DNL the quantity $\frac{t_{0}}{T_{0}}$ equates to a reduction in approximately $49.37 \mathrm{~dB}$ from the purely cumulative SEL values, as represented in Equation (9):

$$
L_{D N}=10 \log \left[\sum_{i=1}^{N} 10^{\left(L_{E, i}+\Delta_{i}\right) / 10}\right]-49.37
$$

with $\Delta_{i}=0$ for day operations and $\Delta_{i}=10$ for night operations. For a given mix of aircraft, where the ratio between each type remains constant and only the number of operations changes, Equation (9) can be replaced by (10):

$$
L_{D N}=\bar{L}_{E}+10 \log \left[N_{\text {day }}+10 N_{\text {night }}\right]-49.37
$$

where $\bar{L}_{E}$ is the operations-energy average SEL value. Consequently, if the average SEL $\left(\bar{L}_{E}\right)$ decreases by a given amount then the DNL will also decrease by a commensurate amount, and the DNL will increase with ten times the log of the number of operations, i.e. a doubling of operations will increase the $L_{D N}$ value at a given point by $3.01 \mathrm{~dB}$.

Since QC relates well to the A weighted SEL ${ }^{10,17,19}$ relationship and is inherently related to actual intensity and not the logarithmic relationship is is reasonable to expect to see 
a good correlation between a weighted sum of QC values and the cumulative measures. Additionally, if cumulative QC formulation correlates well with other proposed means of determining the area exposed to cumulative noise metrics that the case for using $\mathrm{QC}$ as the intermediary design metric could be considered well supported.

\section{IV.B. Correlation Between Cumulative QC and Noise Area Metrics}

The mathematical relationship between single event average noise, SEL, and cumulative average noise, e.g. DNL, provide the first step for determining the effect of a single aircraft on the area exposed to a to a given level of cumulative noise. However, while this relationship seems simple and straightforward, there is the added effect of airport specific routing of aircraft. That is different arrivals and departures will fly different routes into and out of airports. This means that it is not immediately obvious how well a simple weighted summation of $\mathrm{QC}$ will correlate with exposure to cumulative noise.

Dikshit and Crossley ${ }^{4}$ investigated the correlation between a cumulative metric based upon NEE and the area of the $L_{D N}=65 d B$ noise contour, i.e. the area around an airport that is exposed to noise levels equivalent to or greater than $65 d B A$ over a 24 hour period. They found that, in general, it was possible to provide a first order estimate of noise impact using a model, represented in Equation (4), based upon the EPNL certification levels of each aircraft. A similar body of work focusing on the relationship between the QC values and the $S E L$ and UK $L_{e q}$ was performed by the UK CAA as part of the Quota Count review procedure. ${ }^{10,17,19}$

In order to determine if a simple QC based metric could be used to determine variation in $L_{D N}=65 \mathrm{~dB}$ contour area two methods of comparison were undertaken. First, the same D-Optimal design used by Dikshit and Crossley, and given in Tables 5 and 6, was used using the $\mathrm{QC}$ values for the both the representative and 'best-in-class' aircraft. Note: "\% BIC" is

\begin{tabular}{|c|c|c|c|c|c|}
\hline Exp. & \# Pass & \# Cargo & Day Ratio & Fleet mix & $\% \mathrm{BIC}$ \\
\hline 1 & 2 & 2 & 1 & 2 & 1 \\
\hline 2 & 1 & 3 & 1 & 3 & 1 \\
\hline 3 & 2 & 1 & 1 & 1 & 3 \\
\hline 4 & 3 & 3 & 1 & 1 & 2 \\
\hline 5 & 1 & 1 & 3 & 2 & 2 \\
\hline 6 & 1 & 2 & 2 & 1 & 1 \\
\hline 7 & 2 & 1 & 2 & 3 & 2 \\
\hline 8 & 3 & 2 & 3 & 3 & 3 \\
\hline 9 & 2 & 3 & 2 & 2 & 3 \\
\hline 10 & 3 & 1 & 2 & 2 & 1 \\
\hline
\end{tabular}

10 of 22 
percent best in class.

Table 6. Factors and Levels used by Dikshit and Crossley ${ }^{4}$

\begin{tabular}{|c|c|c|c|c|c|}
\hline & ss/day & & Fleet Mix & & \\
\hline 1 & 284 & 1 & $25-2-51-4-1$ & & 1 \\
\hline 2 & 426 & 2 & $18-0-35-13-2$ & & 2 \\
\hline 3 & 568 & 3 & $25-14-49-2-1$ & & 3 \\
\hline & & & & ay 1 & \\
\hline & & & & 0. & 703 \\
\hline & & & s & & 75 \\
\hline & & & : & & 55 \\
\hline
\end{tabular}

Furthermore, to investigate the effect that the cargo and passenger operations have on the resulting response and correlation between the two models two additional treatments were devised. These two treatments kept the same basic formulation, but removed either the passenger or cargo operations respectively. This produced a set of 30 different experiments ranging from 80 to 728 operations per day. The EPNL, NEE and QC values for the representative aircraft that are inputs to these experiments are given in Table 7.

In the first instance the resulting contour area using Equation (4) and the regression coefficients presented in Table 2 were compared to a simple day and night weighted accumulation of QC values of all flights operating out of an airport, using Equation (11).

$$
\begin{aligned}
& Q C_{C u m, D N L}= \\
& \times \sum_{a=1}^{6}\left[\left\{\left(P_{a} \delta_{i}^{\text {dep }}+Q_{a}\left(1-\delta_{i}^{\text {dep }}\right)\right)\left(Q C_{a, r e p}^{\text {pax dep }} X_{a, i, \text { dep }}^{\text {pax,rep }}+Q C_{a, b e s t}^{\text {pax,dep }} X_{a, i, d e p}^{\text {pax }, \text { est }}\right)\right.\right. \\
& \left.+\left(P_{a} \delta_{i}^{a r r}+Q_{a}\left(1-\delta_{i}^{a r r}\right)\right)\left(Q C_{a, r e p}^{\text {pax, arr }} X_{a, i, a r r}^{\text {pax }, \text { rep }}+Q C_{a, b e s t}^{\text {pax, arr }} X_{a, i, a r r}^{\text {pax }, \text { best }}\right)\right\} \\
& +\left\{\left(R_{a} \delta_{i}^{\text {dep }}+S_{a}\left(1-\delta_{i}^{d e p}\right)\right)\left(Q C_{a, \text { rep }}^{\text {car,dep }} X_{a, i, d e p}^{\text {car, rep }}+Q C_{a, b e s t}^{\text {car,dep }} X_{a, i, d e p}^{\text {car, } b e s t}\right)\right. \\
& \left.\left.+\left(R_{a} \delta_{i}^{\text {arr }}+S_{a}\left(1-\delta_{i}^{\text {arr }}\right)\right)\left(Q C_{a, \text { rep }}^{\text {car, }, \text { arr }} X_{a, i, a r r}^{\text {car, rep }}+Q C_{a, \text { best }}^{\text {car,arr }} X_{a, i, a r r}^{\text {car, best }}\right)\right\}\right]
\end{aligned}
$$

The weightings coefficients $\left(P_{a}, Q_{a}, R_{a}, S_{a}\right)$ in Equation (11) are similar to those used in Equation (4). For simple $Q C_{c u m, D N L}$ calculations $P_{a}$ and $R_{a}$ are set to one and $Q_{a}$ and $S_{a}$ are set to 10 to represent the day and night weightings in DNL. The resulting responses showed a generally reasonable, but not perfect correlation between the Area $_{N E E}$ and the $Q C$ based response, as show in Table 8 and Figure 2. The $92 \%$ correlation between the 
Table 7. Noise Response Inputs for Representative Aircraft ${ }^{21}$

\begin{tabular}{ccccccc}
\hline \hline Aircraft & $\left(E P N L_{T O}+\right.$ & $E P N L_{A P}-$ & $Q C_{T O}$ & $Q C_{A P}$ & $Q C_{\text {Cont }, T O}$ & $Q C_{\text {Cont }, A P}$ \\
& $\left.E P N L_{S L}\right) / 2$ & 10 & & & & \\
\hline CRJ 200 & 80.55 & 82.1 & 0 & 0 & 0.113 & 0.203 \\
CRJ 700 & 86.05 & 82.6 & 0.25 & 0 & 0.401 & 0.228 \\
EMB 12022 & 83.1 & 82.7 & 0 & 0 & 0.203 & 0.233 \\
ERJ 145 & 82.95 & 82.5 & 0 & 0 & 0.196 & 0.223 \\
EMB 170 & 88.25 & 85 & 0.5 & 0.25 & 0.665 & 0.396 \\
B727-100QF & 92.2 & 85.3 & 1 & 0.25 & 1.652 & 0.425 \\
B727-200 & 97.95 & 88.1 & 4 & 0.5 & 6.207 & 0.8098 \\
B737-300 & 87.4 & 87.6 & 0.5 & 0.5 & 0.547 & 0.721 \\
B737-700 & 89.4 & 85.8 & 0.5 & 0.25 & 0.867 & 0.476 \\
B737-800 & 92.3 & 86.4 & 1 & 0.5 & 1.690 & 0.547 \\
B757-200 & 90 & 85.2 & 1 & 0.25 & 0.995 & 0.415 \\
B767-300 & 93.6 & 88.5 & 2 & 0.5 & 2.280 & 0.887 \\
B767-300F & 93.6 & 88.8 & 2 & 0.5 & 2.254 & 0.950 \\
A300-600F & 93.7 & 89.9 & 2 & 1.0 & 2.306 & 1.224 \\
A330-200 & 94.4 & 86.9 & 2 & 0.5 & 2.710 & 0.614 \\
B777-200RR & 95.9 & 90 & 2 & 1 & 3.872 & 1.253 \\
B777-200GE & 93.7 & 88.8 & 2 & 0.5 & 2.333 & 0.950 \\
B747-400F & 98.9 & 94.1 & 4 & 2 & 7.725 & 3.221 \\
\hline \hline
\end{tabular}

Table 8. Correlation Between Areanee and QC, DNL Weightings

\begin{tabular}{lccc}
\hline \hline & NEE & QC & $\mathrm{QC}_{\text {cont }}$ \\
\hline $\mathrm{NEE}$ & 1.0000 & 0.9238 & 0.9325 \\
$\mathrm{QC}$ & 0.9238 & 1.0000 & 0.9985 \\
$\mathrm{QC}_{\text {cont }}$ & 0.9325 & 0.9985 & 1.0000 \\
\hline \hline
\end{tabular}




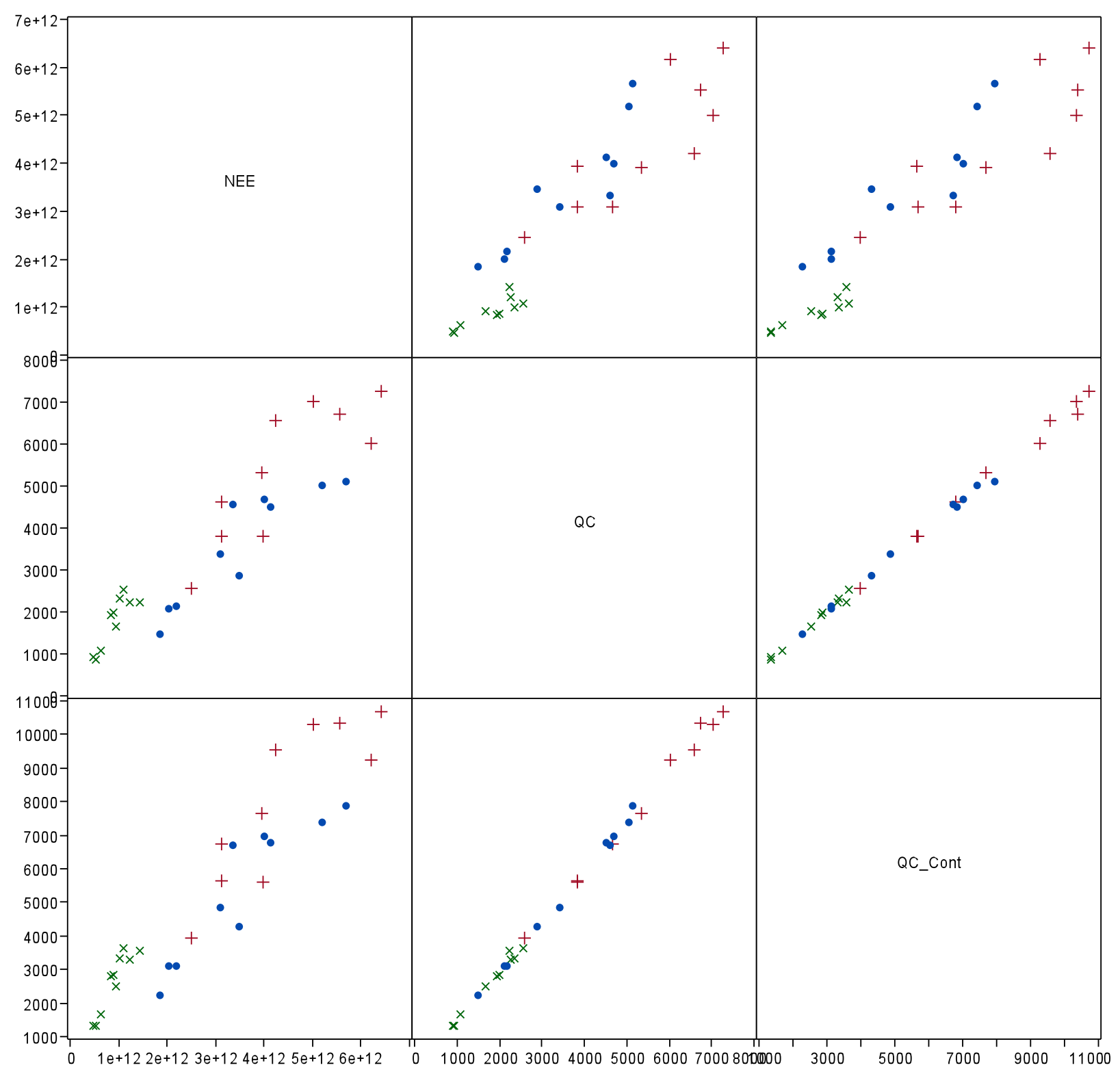

Figure 2. Scatterplot of AreanEE and QC Results, X's represent freight operations only, dots passenger operations only, and pluses all operations.

Presented as paper AIAA-2010-9289 at the 10th Aviation Technology Integration and Operations (ATIO) Conference, Ft Worth, TX, 13-15 September, 2010. 
two responses indicates that the two models should trend very similarly over small ranges, such as those that would be used when performing design trades for a new aircraft system. However, it is potentially useful to attempt to isolate what the cause of the lack of complete correlation might be. This is assisted by looking at the differnt blocks in Figure 2, which isolate the passenger and cargo operations. The result is that some of the operations groups correlate better than others, passenger better than cargo. The first place to look is at the weighting factors, while the DNL weightings are the easiest to use better correlation may be achieved by using the NEE regression coefficents

In order to investigates this the same 30 experiments we rerun, however, the QC accumulation model weightings were replaced by the regression coefficients suggested by Dikshit and Crossley, and given in Table $2 .{ }^{4}$ The results of this gives a much higher correlation, as shown in Table 9 and Figure 3. The result is not at all surprising since the addition of the class

Table 9. Correlation Between AreanEE and QC, using NEE weightings

\begin{tabular}{lccc}
\hline \hline & $\mathrm{NEE}$ & $\mathrm{QC}$ & $\mathrm{QC}_{\text {cont }}$ \\
\hline $\mathrm{NEE}$ & 1.0000 & 0.9978 & 0.9999 \\
$\mathrm{QC}$ & 0.9238 & 1.0000 & 0.9978 \\
$\mathrm{QC}_{\text {cont }}$ & 0.9999 & 0.9978 & 1.0000 \\
\hline \hline
\end{tabular}

specific weightings into Equation (11) produces the same formulation as Equation (4). The slight lack of correlation between the responses of Area $\mathrm{NEE}_{\text {and }} \mathrm{QC}_{\text {cont }}$ and simpler $\mathrm{QC}$ based response is a function of the discrete nature the the QC system. In fact, this limitation of the current QC system was identified by the UK Department for Transport ${ }^{17}$ in 2004. This improved correlation, shows that the simple, day-night weight accumulation of QC values, which performs well overall, does cannot account for all of the variability between aircraft. The issue is that the regression coefficients presented by Dikshit and Crossley are specific to the aircraft types included in the study, reducing their flexibility. The correlations presented in Table 8 are sufficiently high to suggest that the use of simple weighting provides accurate enough results for the increased flexibility and ease of use.

Even though the purpose of the comparison was to determine whether or not QC could reasonably be used to estimate potential changes in cummulative metrics. Because of the result of the simple correlation between the two responses, it is possible to perform a simple transformation of the cumulative QC metric to the contour area estimation provided by the NEE method. This type of transformation represented in Equation (12).

$$
\operatorname{Area}_{N E, e q}=A+\left(B \times Q C_{c u m}\right)
$$




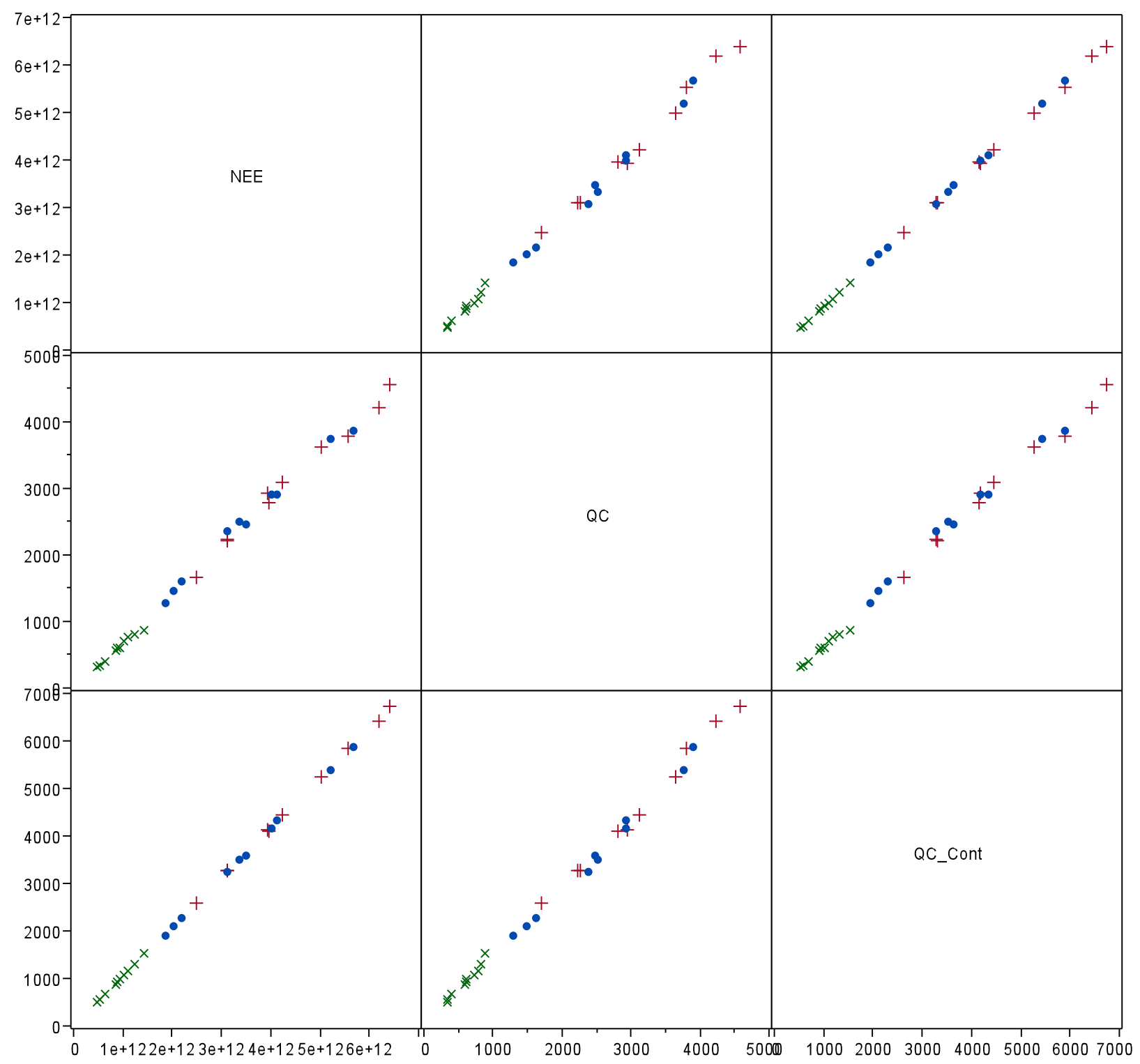

Figure 3. Scatterplot of AreanEE and QC Results, using NEE Weightings. X's represent freight operations only, dots passenger operations only, and pluses all operations.

Presented as paper AIAA-2010-9289 at the 10th Aviation Technology Integration and Operations (ATIO) Conference, Ft Worth, TX, 13-15 September, 2010. 


\section{Design Implications}

While it is one thing to use a simplified area based model for long range environmental benefit projections, such as proposed by Dikshit and Crossley ${ }^{4}$ and Zhao, et al., ${ }^{5}$ it is another thing to use it as a direct design tool. Historically, aircraft and engine manufacturers have relied on the current and potential future certification limitations contained in Annex 16 of the ICAO convention. ${ }^{9}$ This is the now ubiquitous Chapter/Stage 2, 3, 4 scheme. Indeed, a new airplane that does not at least meet the current noise stringency (preferably with a margin) cannot be certified. Further, in most of the world, the US being the primary exception, airports vary their landing fees based upon the aircraft's margin to the current noise regulations. This is one of the reasons that European airlines tended to replace their Chapter/Stage 2 aircraft with new aircraft as the 'phaseout' deadline approach. Conversely US airlines used a cheaper, shorter-term solution of hush kitting and re-certifying their aircraft to just meet the Chapter/Stage 3 regulations. The issue with the certification requirements is that the limit is based upon the certified maximum take-off weight of the aircraft, as show in in Equations (13), (14), and (15). ${ }^{9}$ As such, heavier aircraft are allowed to 'make more noise'.

$$
\begin{gathered}
E P N L_{l i m, F O}=66.65+13.29 \times \log (M T O W / 1000 \mathrm{~kg}) \\
E P N L_{l i m, S L}=80.87+8.51 \times \log (M T O W / 1000 \mathrm{~kg}) \\
E P N L_{l i m, A P}=86.03+7.75 \times \log (M T O W / 1000 \mathrm{~kg})
\end{gathered}
$$

Unfortunately, this has the side effect of partially divorcing the design limitations and goals from the actual benefit that is trying to be achieved, i.e. reducing the actual exposure of people living near and airport to unwanted noise. This was the primary reason for introducing the Quota Count system, first at London area airports, and more recently at a broader spectrum of airports. ${ }^{17}$ The QC system was originally designed to translate into temporal and seasonal limitations in operations that could be assigned to each operator. Further, in many cases the current charging schema at these airports have made use of the $\mathrm{QC}$ values for each aircraft to assess noise surcharges. London's Heathrow, Gatwick, and Stansted airports base their landing fees for Chapter 3 certified aircraft on their QC levels, ${ }^{24-26}$ while Manchester Airport ${ }^{27}$ bases at least some of their landing fees, passenger facility charges, and air traffic service fees on the aircraft's QC level. As Girvin ${ }^{18}$ has indicated many airframe and engine manufacturers have started to use QC as at least a supplemental if not primary aircraft noise design metric ${ }^{\mathrm{a}}$. However, while it is straightforward to use either the QC or

aThe author has had several conversations with engine and airframe design engineers which support Girvin's statement. Further, there is at least anecdotal evidence that the design of the A380 was influenced by Heathrow's QC operations limits. 
a continuous equivalent of QC to evaluate an aircraft's absolute noise performance, it is somewhat less so if one is trying to compare two significantly different aircraft or trade noise with one of many other metrics.

Regardless of the next level of metric, any improvement in the noise level of aircraft should reduce the number of people exposed to harmful or annoying noise. While there is dispute over what the relationship is between noise and human health along with dispute over what are the most appropriate measures of noise, ${ }^{28}$ the most common measures are those of the A-weighted equivalent, time-average variety, e.g. $\mathrm{L}_{\mathrm{eq}} / \mathrm{LA}_{\mathrm{eq}}$ or $\mathrm{L}_{\mathrm{DN}}$, and the number of people exposed to specific values of these measures. The issue with using people exposed to a given level of $\mathrm{L}_{e q}$ or $\mathrm{L}_{\mathrm{DN}}$ is that the relationship between the area contained within a specific contour and the populaiton exposed tends to be highly non-linear. ${ }^{4}$ It is for this reason that Dikshit and Crossley focus on reducing the area within and not the population exposed to $L_{D N}=65 \mathrm{dBA}$. Consequently several organizations are developing sophisticated models to calculate the population exposure and ultimately actual damage. ${ }^{3,29,30}$

Again this issue is one that is more prominent when looking at long-term technology trends and not specific aircraft designs. If the primary goal is to compare the noise performance of two designs then it is actually the difference in the noise exposure, either in terms of contour area or number of people exposed that is of the most interest. As demonstrated by Dikshit and Crossley, there is a reasonable correlation between the NEE metric and the area contained withing the $65 \mathrm{~dB}$ DNL contour, ${ }^{4}$ and the QC level matches well with the relative size of the $90 d B$ SEL contour. ${ }^{10,17}$ This work has shown that a reasonable correlation exists between cumulative QC levels and the resulting NEE estimate $65 \mathrm{~dB}$ contour area. However, the only way to match the NEE area estimation values with INM determined contour area was to implement a series of day and night coefficients, shown in Table 2, that varied between aircraft class and operation type, which is not conducive to a simple design metric. It would be much more convenient to use the standard operations weighting coefficients for the appropriate $\mathrm{L}_{\mathrm{eq}}$ value. Since the idea is to use a single set of aircraft related values as a extensive metric, the use of several different $\mathrm{QC}_{\text {cum }}$ values is still not ideal.

There is, however, the benefit that design choices typically compare two slightly different designs. Further, as the design progresses the absolute difference between two design options will decrease. This means that instead of investigating absolute correlation with area, and ultimately population exposed, we only need to compare the difference in the area or change in area. The benefit of this is that while the correlation may fall down using different weighting metrics, especially for a broad range of aircraft types and operations, it should be much better when comparing the deltas.

As a test, the correlation between the absolute and percent different in AreaneE and $\mathrm{QC}_{\mathrm{Cum}}$ for an new aircraft design in classes 3 and 4 was investigated. This simple test case 
was chosen as these two classes made up the largest portion of the operations in Dikshit and Crossley's analysis, ${ }^{4}$ and they are represented by two different families of aircraft, Boeing's 737 and Airbus's A320 families. Furthermore, this class of aircraft is commonly thought to be the next major new aircraft development program. This study was performed using the same design of experiments given in Tables 5 and 6, using the same three treatments shown in the previous section. The only significant changes were that instead of the representative aircraft forming the baseline, all operations used only the quietest aircraft listed in Table 7 for each class and the prior best-in-class aircraft in classes 3 and 4 were replaced by a new, notional aircraft. The primary reason for using only the quietest aircraft is that some of the other classes of aircraft are extremely loud, one only has to look at the QC values for the Boeing 727-200 and 747-400F, and tend to swamp the benefits achieved through the use of quieter aircraft.

The notional replacement aircraft in seat classes 3 and 4 were assumed to have cumulative EPNL values 20dB quieter than the current aircraft, the Boeing 737-700 and 737-800. The individual certification points were apportioned as $8 \mathrm{~dB}$ each for takeoff/flyover and sideline and $4 \mathrm{~dB}$ for approach. This corresponds to the relative difficulty of reducing approach noise signatures. The resulting pseudo-certification/NEE values and the corresponding QC values are given in Table 10. One issue that arises from the reduced noise is that both of the

Table 10. Noise Response for Notional Replacement Aircraft

\begin{tabular}{ccccccc}
\hline \hline Aircraft & $\begin{array}{c}\left(E P N L_{T O}+\right. \\
\left.E P N L_{S L}\right) / 2\end{array}$ & $\begin{array}{c}E P N L_{A P}- \\
10\end{array}$ & $Q C_{T O}$ & $Q C_{A P}$ & $Q C_{C o n t, T O}$ & $Q C_{C o n t, A P}$ \\
\hline RA3 & 81.4 & 81.8 & 0 & 0 & 0.138 & 0.190 \\
RA4 & 84.3 & 82.4 & 0.25 & 0 & 0.268 & 0.218 \\
\hline \hline
\end{tabular}

notional aircraft have at least one $\mathrm{QC}$ value that fall below the minimum measured response of $84 \mathrm{~dB}$. This means they will produce no net effect on the metrics that use the raw QC as a response, potentially overstating their benefit.

The resulting calculations were done using the weightings in Table 2 for the NEE based area calculations and the simple day night weighting for the cumulative QC response. The resulting correlation between the percent difference in the cumulative metrics is given in Table 11. The correlation remains reasonable for large differences in the noise level between

Table 11. Correlation Between the \% Differenes for AreaneE and $\mathrm{QC}_{\mathrm{Cum}}$

\begin{tabular}{lccc}
\hline \hline & $\mathrm{NEE}$ & $\mathrm{QC}$ & $\mathrm{QC}_{\text {cont }}$ \\
\hline $\mathrm{NEE}$ & 1.0000 & 0.9818 & 0.9906 \\
$\mathrm{QC}$ & 0.9818 & 1.0000 & 0.9953 \\
$\mathrm{QC}_{\text {cont }}$ & 0.9906 & 0.9953 & 1.0000 \\
\hline \hline
\end{tabular}


the two aircraft. In the case where the $\mathrm{QC}$ values of the notional aircraft go to zero, the results with the $\mathrm{QC}_{\text {cum }}$ mark over predict the reduction in impact, especially for the cases that have a high percentage of seat class 3 and seat class 4 operations. This is still present in the case where $\mathrm{QC}_{\text {Cont,Cum }}$ is used instead, but the effect is much smaller. This is shown in Figure 4. Note: the highlighted points, triangles, are cases where the operations of the

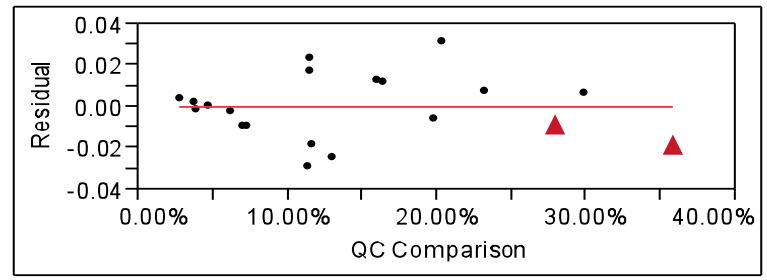

(a) $\mathrm{QC}_{\text {cum }}$

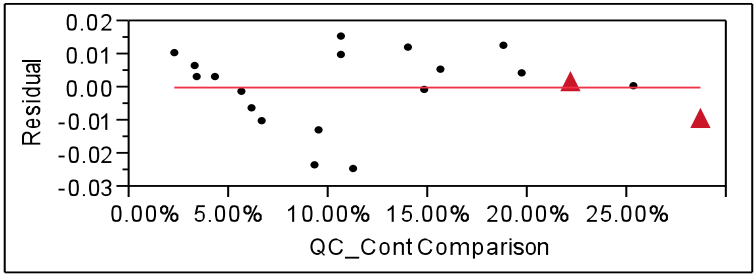

(b) $\mathrm{QC}_{\text {Cont,Cum }}$

Figure 4. Residual for Bivariate Fit of QC cumulative response vs. NEE Area Estimate Percent Difference

notional seat class 3 and seat class 4 aircraft dominate.

It should be noted that this is an estimate of the total difference between an existing system and that of a new system. As mentioned above in most cases design choices involve significantly smaller differences, and the correlations and applicability should be correspondingly better.

While the correlations shown in Table 11 and Figure 4 applied to the relationship between two different metrics with two differing weighting schema that ultimately relate to the area exposed to greater than $L_{D N}=65 d B$, it is reasonable to apply them to other metrics, such as the UK's $\mathrm{L}_{\mathrm{eq}}$ or the less common $\mathrm{L}_{\mathrm{DEN}}$ as the weightings are relatively well known and difference time constants result in a simple displacement from the origin, as shown in Equations (8), (9), and (10). This indicates that while QC is a relatively straightforward and easy measure to communicate the noise performance of an aircraft there are issues with the current exemption level, i.e. below $Q C=0.25$ and the noncontinuous nature of the $\mathrm{QC}$ system. As such it has been recommended, ${ }^{17}$ and is the authors' opinion, that a continuous form of QC be used as the measure for computing noise performance. This can be converted into a standard QC value for outside reporting if desired. A simple formulation of this 
continuous value for the current QC scale is represented in Equation (16).

$$
Q C_{\text {cont }}=\frac{\left[\frac{10^{(E P N L / 10)}}{10^{8.4}}\right]}{4}
$$

The final issue is the use of QC in general and changes in an aircraft's QC response as a measure of noise performance is the relationship between the QC value and the actual number of people exposed to annoying or harmful levels of noise. As mentioned before the non-linear relationship between contour area and exposure are well understood and several groups are investigating how to simplify this process. However, when dealing with a choice between two design choices the differences in $\mathrm{QC}$ or $\mathrm{QC}_{\text {cont }}$ are quite small, generally much smaller than the $20 \mathrm{~dB}$ used in the above example. Consequently, the resulting change in contour area will be correspondingly smaller. As such it is relatively reasonable to assume the change population exposure, across many airports, should be relatively small and nearly linear. This assumption may not hold and it is imperative that future research into the effect of noise exposure be followed closely.

\section{Conclusions}

The increased focus on aircraft environmental effects has lead to the development of simplified measures for estimating the direct impact of a single aircraft operation on the local and global environment. One aspect of this was the development of the QC system in the United Kingdom. Because the QC system works on the absolute and not weight adjusted certification noise values, as compared to the certification standard, it is a better measure of the actual impact. Prior work relating QC to single even metrics and straightforward fleet models based upon certification noise values plus the strong correlation between the simple additive QC and NEE models indicate that the QC is a very good surrogate for the actual, predicted impact of a single aircraft or aircraft in a fleet. As such it provides a reasonably good ext ensive metric that can be used when comparing different aircraft design alternatives and their broader noise impact. It is accessible to the design engineer using the current design noise analysis tools and relatively easy to communicate with the outside community. An aircraft with a QC value of 1 has one-half the single event and cumulative impact of an aircraft with a QC value of 2. This is something that is intelligible and accessible to both the design and analysis engineers and the broader public. Using QC as a design metric in place of all of the other noise measures, therefore, provides a single, accessible measure to both engineering decision makers and outside stakeholders. 


\section{References}

${ }^{1}$ Collopy, P. D., "A System for Values, Communication, and Leadership in Product Design," 1996 Internationl Powered Lift Conference, No. 96-2287, SAE International, November 1996. doi:10.4271/ 962287.

${ }^{2}$ Collopy, P. D., "Surplus Value in Propulsion System Design Optimization," 33rd AIAA/ASME/SAE/ASEE Joint Propulsion Conference and Exhibit, No. 97-3159, Seattle, WA, July 6-9 1997.

${ }^{3}$ Waitz, I., Lukachko, S., Go, Y., Hollingsworth, P., Harback, K., and Morser, F., "Requirements Document for the Aviation Environmental Portfolio Management Tool," Tech. Rep. PARTNER-COE-2006001, Partnership for AiR Transportation Noise and Emissions Reduction, Cambridge, MA, 2006.

${ }^{4}$ Dikshit, P. N. and Crossley, W. A., "Airport Noise Model Suitable for Fleet Studies," 9th AIAA Aviation Technology, Integration, and Operations (ATIO) Conference, No. AIAA-2009-6937, Hilton Head, SC, 21-23 September 2009.

${ }^{5}$ Zhao, J., Tetzloff, I. J., Tyagi, A., Dikshit, P., Mane, M., Agusdinata, D., Crossley, W. A., and DeLaurentis, D., "Assessing New Aircraft and Technology Impacts on Fleet-Wide Environmental Metrics including Future Scenarios," 48th AIAA Aerospace Sciences Meeting Including The New Horizons Forum and Aerospace Exposition, No. AIAA-2010-282, American Institute of Aeronautics and Astronautics, Orlando, FL, 4-7 January 2010.

${ }^{6}$ Pfänder, H., Hollingsworth, P., Jimenez, H., and Won, H., "Environmental Challenge: How to Close the Gap Between Policy and Technology?" 9th AIAA Aviation Technology, Integration, and Operations (ATIO) Conference, No. AIAA-2009-7003, Hilton Head, SC, 21-23 September 2009.

${ }^{7}$ Jimenez, H., Pfaender, H., and Mavris, D., "Environmental Impact Analysis of Fleet and Policy Options of Aircraft Operators using System Dynamics," 10th AIAA Aviation Technology, Integration, and Operations (ATIO) Conference, No. AIAA-2010-9251, American Institute of Aeronautics and Astronautics, Fort Worth, TX, 13-15 September 2010 [cited 13 March 2011].

${ }^{8}$ FAA, "Title 14 Aeronautics and Space - Code of Federal Regulations, Part 36, Appendix B," Regulation 14CFR Part 36, Federal Aviation Administraiton, Washington, DC, 2011 [cited 13 March 2011].

9 "ICAO Annex 16 Volume I Aircraft Noise," Policy, International Civil Aviation Organization, Montreal, Canada [cited January 30, 2009].

${ }^{10}$ Ollerhead, J. B. and Hopewell, H., "Review of the Quota Count (QC) System: Re-Analysis of the Difference between Arrivals and Departures," ERCD Report 0204, Environmental Research and Consultancy Department, Directorate of Airspace Policy, Civil Aviation Authority, London, UK, 2002.

${ }^{11}$ CAEP, "Assessing Current Scientific Knowledge, Uncertainties and Gaps in Quanitfying Climate Change, Noise and Air Quality Aviation Impacts," Tech. rep., International Civil Aviation Organization, Committee on Aviation Environmental Protection, Washington, DC and Manchester, October-November 2009 [cited 13 March 2011].

${ }^{12}$ Finegold, L., "Sleep disturbance due to aircraft noise exposure," Noise and Health, Vol. 12, No. 47, 2010, pp. 88-94. doi:10.4103/1463-1741.63208.

13 "Integrated Noise Model," [online]. Federal Aviation Administraiton, Washington, DC, 2006. Available from: http://www.faa.gov/about/office_org/headquarters_offices/aep/models/inm_model/ [cited 28 July 2010]. 
${ }^{14}$ Rhodes, D. P., "Updated Methodology and Supplementary Information Relating to Future Aircraft Noise Exposure Estimates for UK Airports," ERCD Report 0307, Environmental Research and Consultancy Department, Civil Aviation Authority, London, UK, December 2003.

15 "Night Flights at Heathrow, Gatwick and Stansted Airports: Proposals for revised restrictions from 24 October 1993," Consultation paper, Department of Transport, London, UK, January 1993.

${ }^{16}$ Butcher, L., "Aviation: Night Flights," Standard Note SN/BN/1252, United Kingdom House of Commons Library, London, UK, 26 May 2010.

17 "Review of Quota Count (QC) System used for Administration of Night Noise Quotas at Heathrow, Gatwick and Stanstead Airports," Tech. rep., Department of Transport, 2004.

${ }^{18}$ Girvin, R., "Aircraft noise-abatement and mitigation strategies," Journal of Air Transport Management, Vol. 15, No. 1, Jan. 2009, pp. 14-22. doi:10.1016/j.jairtraman.2009.02.012.

${ }^{19}$ White, S., Ollerhead, J. B., Cadoux, R. E., and Smith, M. J. T., "Quota Count Validation Study: Noise Measurements and Analysis," ERCD REPORT 0205, Environmental Research and Consultancy Department, UK Civil Aviation Authority, Norwich NR3 1GN, 2003.

${ }^{20}$ ECAC.CEAC Doc 29: Report on Standard Method of Computing Noise Contours Around Civil Airports, Vol. 1, European Civil Aviation Conference, 3rd ed., December 2005.

21 "EASA approved noise levels for jet aeroplanes Type Certificate Data Sheet jets (Issue 8)," Excel Spreadsheet, 26 April 2010 [cited 26 July 2010].

22 "EASA approved noise levels for heavy propeller driven aeroplanes Type Certificate Data Sheet heavy props (Issue 7)," Excel Spreadsheet, 16 December 2009 [cited 26 July 2010].

23 "Noise Levels for U.S. Certificated and Foreign Aircraft," Advisory Circular AC 36-1H, Federal Aviation Administration, Washington, DC, 15 November 2001.

24 "Heathrow Airport: Conditions of Use Including Airport Charges from 1 April 2010," Conditions of use, Heathrow Airport Limited, Hounslow, Middlesex, TW6 1JH, United Kingdom, 29 March 2010.

25 "Gatwick Airport: Conditions of Use, Including Airport Charges from 1 April 2010," Conditions of use, Gatwick Airport Limited, West Sussex, RH6 0NP, United Kingdom, 23 February 2010.

26 "Stansted Airport: Conditions of Use, Including Airport Charges from 1 April 2010," Conditions of use, Stansted Airport Limited, Stansted, Essex, CM14 1QW, United Kingdom, 1 March 2010.

27 "Manchester Airport PLC: Schedule of Charges and Terms and Conditions of Use 1 April 2010 to 31 March 2011," Conditions of use, Manchester Airport PLC, Manchester, M90 1QX, United Kingdom, 12 March 2010.

${ }^{28}$ Swift, H., "A Review of the Literature Related to Potential Health Effects of Aircraft Noise," Report PARTNER-COE-2010-003, Partnership for Air Transportation Noise and Emissions Reduction, Cambridge, MA 02139, USA, July 2010.

${ }^{29}$ Fleming, G. G., "Aviation Environmental Design Tool (AEDT) Overview," Tech. rep., U. S. Department of Transportation, Volpe Transportations Systems Center, Cambridge, MA, December 2006.

${ }^{30}$ Waitz, I., Lukachko, S., Willcox, K., Belobaba, P., Garcia, E., Hollingsworth, P., Mavris, D., Harback, K., Morser, F., and Steinbach, M., "Architecture Study for the Aviation Environmental Portfolio Management Tool," Tech. Rep. PARTNER-COE-2006-002, Partnership for AiR Transportation Noise and Emissions Reduction, Cambridge, MA, 2006. 